\title{
BILATERAL ORGANIZATION OF THE VOCAL CONTROL PATHWAY IN THE BUDGERIGAR, MELOPSITTACUS UNDULATUS ${ }^{1}$
}

\author{
JOHN A. PATON, ${ }^{2}$ KIRK R. MANOGUE, AND FERNANDO NOTTEBOHM
}

The Rockefeller University, New York, New York 10021

\begin{abstract}
Anatomical and electrophysiological methods were used to map the vocal control nuclei of the budgerigar, Melopsittacus undulatus. Beginning with the motor nucleus of the syrinx, nuclei were located using antidromic stimulation and then injected with horseradish peroxidase (HRP). Retrogradely transported HRP labeled afferents to the injected nucleus. This procedure was repeated at successively higher levels along the vocal pathway. Connections found using this strategy then were confirmed using anterograde transport of HRP and/or tritiated proline and orthodromic electrical stimulation.

We found that the primary vocal control pathway consisted of (1) the motor nucleus innervating the trachea and syrinx, nXIIts; (2) an archistriatal nucleus, RA; and (3) a neostriatal nucleus, "HVc." These nuclei correspond to similar, possibly homologous, nuclei in the vocal control pathway of the canary (Nottebohm, F., T. M. Stokes, and C. M. Leonard (1976) J. Comp. Neurol. 165: 457-486) but, because of differences in gross brain morphology, are displaced considerably in absolute position. Furthermore, the projection from RA to the motor nucleus is bilateral in the budgerigar, whereas the same connection is strictly ipsilateral in the canary. The projection of the motor nucleus to muscles of the vocal organ is also bilateral in the budgerigar (Manogue, K. R., and F. Nottebohm (1981) J. Comp. Neurol., in press) but ipsilateral in the canary. The possible significance of these species differences for lateralization of motor control is discussed.
\end{abstract}

Vocal control is lateralized in the several species of oscine songbirds examined to date. At the periphery, section of the left hypoglossal nerve innervating the left side of the avian vocal organ, the syrinx, leads to the loss of a majority of song elements, whereas cutting the right nerve causes a loss of the complementary, much smaller set of elements (Nottebohm, 1971; Lemon, 1973; Nottebohm and Nottebohm, 1976; Seller, 1979).

A form of lateralization also occurs in the brain pathway which controls the syrinx. In canaries, the essential elements of this pathway include the hyperstriatum ventrale pars caudale (HVc), which projects to the robust nucleus of the archistriatum (RA), which in turn projects to the caudal half of the hypoglossal nucleus (nXIIts), formed by the motor neurons that innervate the muscles of the trachea and syrinx. Lesions of the left HVc cause a much greater deficit in the number of song elements

\footnotetext{
' This research was supported by postdoctoral funds from Training Grant 1512 from the National Institute of Mental Health to The Rockefeller University and United States Public Health Scrvice Postdoctoral Fellowship 1F32MH08231 to K. R. M., National Institute of Mental Health Grant 5R01MH18343 to F. N., and National Science Foundation Grant BNS7924602 to J. A. P. We would like to thank Darcy Kelley for sharing her experience and enthusiasm with us.

${ }^{2}$ To whom correspondence should be addressed at The Rockefeller University, 1230 York Avenue, New York, NY 10021.
}

and in the overall stereotypy of the song pattern than do comparable lesions of the right HVc (Nottebohm, 1977). This hemispheric dominance is of general interest since lateralization of function has received so much attention in the study of the human brain, and in particular, with respect to speech and language skills (reviewed in Geschwind, 1970; Gazzaniga, 1970; Sperry, 1974; Milner, 1974; Gazzaniga and LeDoux, 1978; Nottebohm, 1979).

The vocal system in the order Psittaciformes (parrots and their relatives) is quite different from that of songbirds, at least at the peripheral level. For example, in the parrot Amazona amazonica, cutting the right or left hypoglossal nerve causes little or no change in stereotyped vocalizations (Nottebohm, 1976). This result appears to be due to the fact that axons from each right and left nXIIts innervate the muscles on both right and left syringeal halves as demonstrated in another psittaciform, the budgerigar (Manogue and Nottebohm, 1981).

Given the lack of lateralization in the periphery, does the central nervous system of psittaciforms show hemispheric dominance? To answer this question, the vocal pathway first must be identified. In this paper, we report that the vocal pathway in the budgerigar contains a number of nuclei which are similar or even homologous to nuclei in the canary pathway but that these nuclei are 
displaced relative to their positions in the canary brain. In addition, one pathway which is strictly unilateral in the canary is bilateral in the budgerigar. A portion of these results have been presented in abstract form (Paton et al., 1980).

\section{Materials and Methods}

We used adult budgerigars (Melopsittacus undulatus), both males and females, purchased from Canary Bird Farm, Old Bridge, NJ. During surgery and electrophysiology, animals were anesthetized with $0.0033 \mathrm{ml} / \mathrm{gm}$ of body weight of Chloropent (Fort Dodge Laboratories) injected into the pectoral muscles. The initial dose was supplemented by $0.015-\mathrm{ml}$ injections at $45-\mathrm{min}$ intervals.

During surgery, the birds were fixed in a stereotaxic frame by ear bars and a rod between the mandibles. In the budgerigar, both mandibles are hinged, offering a poor reference for setting the brain case at a reproducible angle. As a result, rostrocaudal and depth coordinates varied from animal to animal. For this reason, electrodes and injections were placed in the brain using electrophysiological methods rather than stereotaxic coordinates alone.

The brain was exposed by opening a slit in the scalp, cutting the thin layers of the skull away with a scalpel, and then removing the dura with fine forceps or iridectomy scissors. The exposed brain was kept moist with saline.

The nerves leading to the syrinx were exposed by rotating the body ventral side up and cutting a rostrocaudal slit in the skin over the trachea. The tracheosyringeal nerve on the near side of the trachea was dissected free, placed on a pair of hook electrodes, and covered with a pool of mineral oil. The hook electrodes were used for stimulation and, at other times, for recording. The nerve was stimulated with a bipolar pulse $(0.3 \mathrm{msec}$ of one polarity followed by $0.1 \mathrm{msec}$ of the other polarity) whose component amplitudes were adjusted to minimize stimulus artifacts recorded in nXIIts.

In the central nervous system, resin-coated tungsten electrodes ( 1 to 2 megohms, Bak) were used for either recording or stimulation. The electrodes were mounted on microdrives attached to the stereotaxic frame and advanced manually with the microdrives or remotely using a Narashige hydraulic microdrive. Cathodal 0.3msec pulses of constant current $(\leq 150 \mu \mathrm{A})$ were used for stimulation, either as isolated pulses or in trains with a 50-msec interpulse interval.

All recordings were amplified 1000 times with $\mathrm{AC}$ coupled differential preamplifiers. Stimulus artifact was suppressed in some recordings by a voltage-controlled amplifier whose gain was set to 0 during the stimulus pulse. Recordings were monitored with an audio monitor and a storage oscilloscope.

Horseradish peroxidase (HRP, Boehringer Mannheim, grade I) was used as both an anterograde and retrograde label for tracing neural pathways. HRP solution (25\% HRP in $0.05 \mathrm{M}$ Tris (tris(hydroxymethyl)aminomethane) at $\mathrm{pH}$ 7.6) was iontophoresed into the brain from micropipettes with a tip size of 20 to $30 \mu \mathrm{m}$. Anodal currents of 1 to $3 \mu \mathrm{A}$ for 10 to $20 \mathrm{~min}$ produced injection sites several hundred micrometers in diameter.
After HRP injection, wounds were sutured closed and the birds were allowed to recover for 24 to $48 \mathrm{hr}$. At that time, the birds were given an overdose of Chloropent and perfused transcardially with $60 \mathrm{ml}$ of $0.9 \% \mathrm{NaCl}$ followed by $60 \mathrm{ml}$ of a glutaraldehyde fixative $(2.5 \%$ glutaraldehyde, $1 \%$ dimethyl sulfoxide, $5 \%$ sucrose in $0.1 \mathrm{M}$ phosphate buffer, $\mathrm{pH}$ 7.6). The brains were removed from the skull and placed in fixative with a sucrose concentration of $30 \%$. After $48 \mathrm{hr}$ at $4^{\circ} \mathrm{C}$, the brains were embedded in gelatin/albumin, frozen, and cut into six series of $50-\mu \mathrm{m}$ sections. Sections were stored in $0.1 \mathrm{~m}$ phosphate buffer at $4^{\circ} \mathrm{C}$ or in a solution containing $30 \%$ sucrose and $30 \%$ ethylene glycol in $0.1 \mathrm{M}$ phosphate buffer at $-10^{\circ} \mathrm{C}$.

Sections were processed for HRP using either $3,3^{\prime}, 5,5^{\prime}$ tetramethylbenzidine (TMB, Sigma) for greatest sensitivity or 3,3'-diaminobenzidine (DAB, Sigma, grade II) for well defined morphology of labeled cells. The protocol for TMB processing followed that of Mesulam (1978) and is described by Manogue and Nottebohm (1981). The protocol for DAB processing was taken from Malmgren and Olsson (1978) and modified by including a preincubation step in cobalt solution (Adams, 1977) as described by Manogue and Nottebohm (1981). Sections were transferred onto chrome alum-coated slides from a $1 \%$ gelatin or a gelatin/alcohol mounting solution. The slides were dried, counterstained, and coverslipped with Permount (Fisher). The TMB sections were counterstained with either neutral red or thionin (Adams, 1980); the DAB sections were counterstained with cresyl violet.

Proline was used as an anterograde label in one animal. Concentrated tritiated proline $(10 \mathrm{mCi} / \mathrm{ml}$, New England Nuclear, TRK534) was iontophoresed from a micropipette (25- $\mu \mathrm{m}$ tip) into RA. After $24 \mathrm{hr}$, the bird was killed and perfused with saline followed by formol/saline, and the brain then was processed for amino acid autoradiography using standard methods (see Kelley and Nottebohm (1979) for details).

\section{Results}

Our strategy for determining the vocal pathway in the budgerigar was to begin at the periphery and work centrally using antidromic stimulation to place HRP at successively higher levels until the telencephalon was reached. Figure 1 shows all of the electrode positions used in these experiments. Anterograde transport of HRP or tritiated proline from the same locations was used to confirm the retrograde results and as a control for the spread of HRP from the injection sites. Orthodromic stimulation then was used (at each level) to confirm the potential control of the syrinx by the nuclei of the vocal pathway. Where budgerigar nuclei are similar to those in songbirds based on the criteria of (1) cell morphology and packing, (2) shape of the nucleus, (3) apposition to identifiable lamina or nuclei, (4) anatomical connections to other nuclei, and (5) function as indicated by the results of microstimulation tests, we refer to these nuclei by the names given to similar nuclei in the canary (Nottebohm et al., 1976; Kelley and Nottebohm, 1979; F. N. Nottebohm, D. B. Kelley, and J. A. Paton, submitted for publication).

Afferents to syringeal motor neurons. The motor nucleus of the syrinx, nXIIts, was found in six birds by advancing a tungsten or HRP-filled micropipette through 


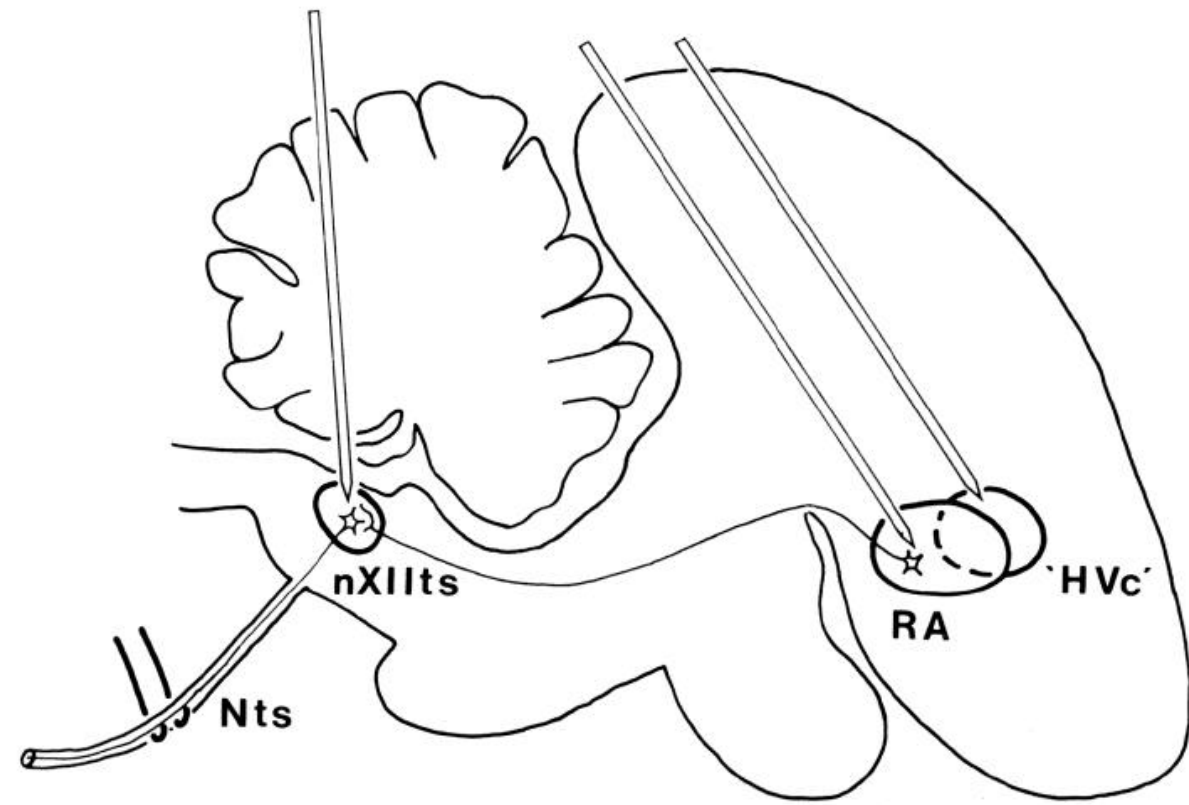

Figure $1 .^{3}$ Schematic diagram of budgerigar brain in a side view. The vocal control nuclei, "HVc," RA, and nXIIts, are shown as are the electrode positions used in antidromic and orthodromic stimulation and recording.

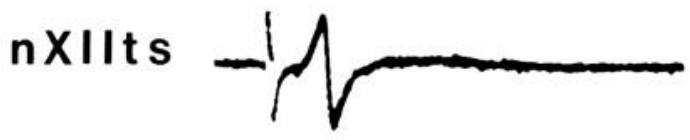

Nts

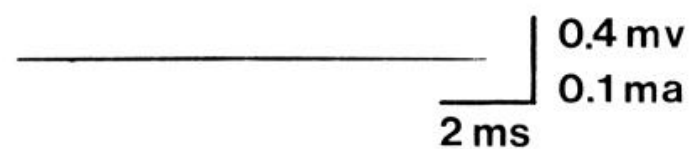

Figure 2. Evoked potentials (five trials) recorded in nXIIts in response to electrical stimulation of the tracheosyringeal nerve, $\mathrm{Nts}$.

the cerebellum and medulla until an antidromically evoked potential was recorded in response to stimulation of the tracheosyringeal nerve (Fig. 2). Iontophoresis of HRP into nXIIts resulted in the labeling of fibers and cell bodies in the nearby medulla, in the midbrain, and in the archistriatum as shown in Figure 3.

In the medulla, fibers containing HRP could be traced from the lateral edges of nXIIts into longitudinal tracts extending rostrally to join the occipitomesencephalic tract (Fig. $3 a$ ). Labeled cells and fibers were seen within the ventrolateral medulla, but because of their proximity to the injection site, they may have been labeled by extracellular diffusion of HRP.

\footnotetext{
${ }^{3}$ The abbreviations used on the figures are: $\mathrm{Cb}$, cerebellum; $\mathrm{CoA}$, commissura anterior; DIP, nucleus dorsalis intermedius posterior thalami; DM, nucleus intercollicularis pars dorsalis medialis; $\mathrm{Hb}$, habenula; $\mathrm{HVc}$, hyperstriatum ventrale pars caudalis; LAD, lamina archistriatalis dorsalis; LMD, lamina medullaris dorsalis; MAN, nucleus neostriatalis anterior magnocellularis; MLd, nucleus mesencephalicus lateralis pars dorsalis; $\mathrm{N}$, neostriatum; na, nucleus ambiguus; $\mathrm{Nts}$, nervus tracheosyringealis; nXIIts, nucleus nervi hypoglossi pars tracheosyringealis; OM, tractus occipitomesencephalicus; RA, nucleus robustus archistriatalis; $\mathrm{TeO}$, tectum opticum; V, ventriculus; vh, ventral horn of spinal cord.
}

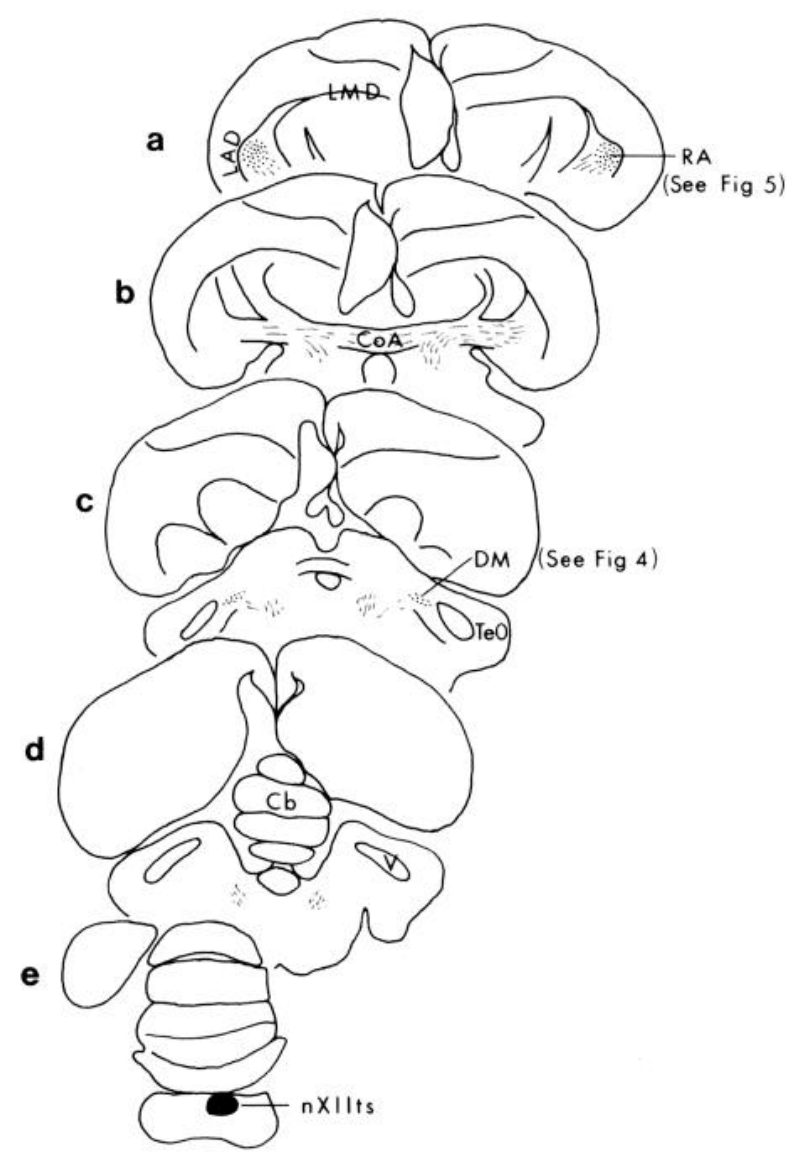

Figure 3. Schematic reconstruction from a transverse section of the brain showing areas labeled after injection of HRP into the motor nucleus, nXIIts. Sections are named from rostral $(a)$ to caudal $(e)$. The injection site is black, representative labeled fibers appear as lines, and representative labeled cells appear as dots. Photomicrographs of DM and RA in this brain are shown in Figures 4 and 5, respectively. 
In the midbrain, some fibers leave the occipitomesencephalic tract and enter a small teardrop-shaped nucleus dorsomedial to the nucleus mesencephalicus lateralis pars dorsalis (see Fig. $3 c$ ). This small nucleus is a subdivision of the nucleus intercollicularis and thus is known as the dorsomedial nucleus of the nucleus intercollicularis (DM; Gurney and Konishi, 1979). The cells of DM have oblong multipolar somas (averaging $12 \times 29 \mu \mathrm{m}, n=20$ cells in one DM) oriented along the longer dorsomedial to ventrolateral axis of the nucleus (Fig. $4 b$ ). The processes of labeled cells in DM tended to be oriented along the same axis.

Most labeled fibers in the occipitomesencephalic tract continued rostrally to the level of the thalamus where they either crossed the midline in the anterior commissure or turned laterally into the telencephalon (Fig. $3 b$ ). On both sides, the fibers ended in an ovoid nucleus delineated by surrounding fiber tracts: the lamina archi- striatalis dorsalis on the dorsolateral side and fiber bundles from the occipitomesencephalic tract on the medial side (Figs. $3 a$ and $5 a$ ). Many of the loosely packed, 11$\mu \mathrm{m}$-diameter cells (average of 20 cells in one RA) within this nucleus contained granular label (Fig. $5 b$ ). Based on its location within the archistriatum, its apposition to lamina archistriatalis dorsalis, its morphology and distribution of cells, and its projection to nXIIts, we identified this nucleus as the nucleus robustus of the archistriatum (RA).

In summary, unilateral injections of the motor nucleus led to bilateral labeling of fibers in the occipitomesencephalic tract and the anterior commissure, and bilateral labeling of cells in DM and RA.

Afferents and efferents of RA. We located RA in nine birds by finding the motor nucleus as before, stimulating RA terminals in the motor nucleus, and searching for antidromic responses in the general area of the telen-

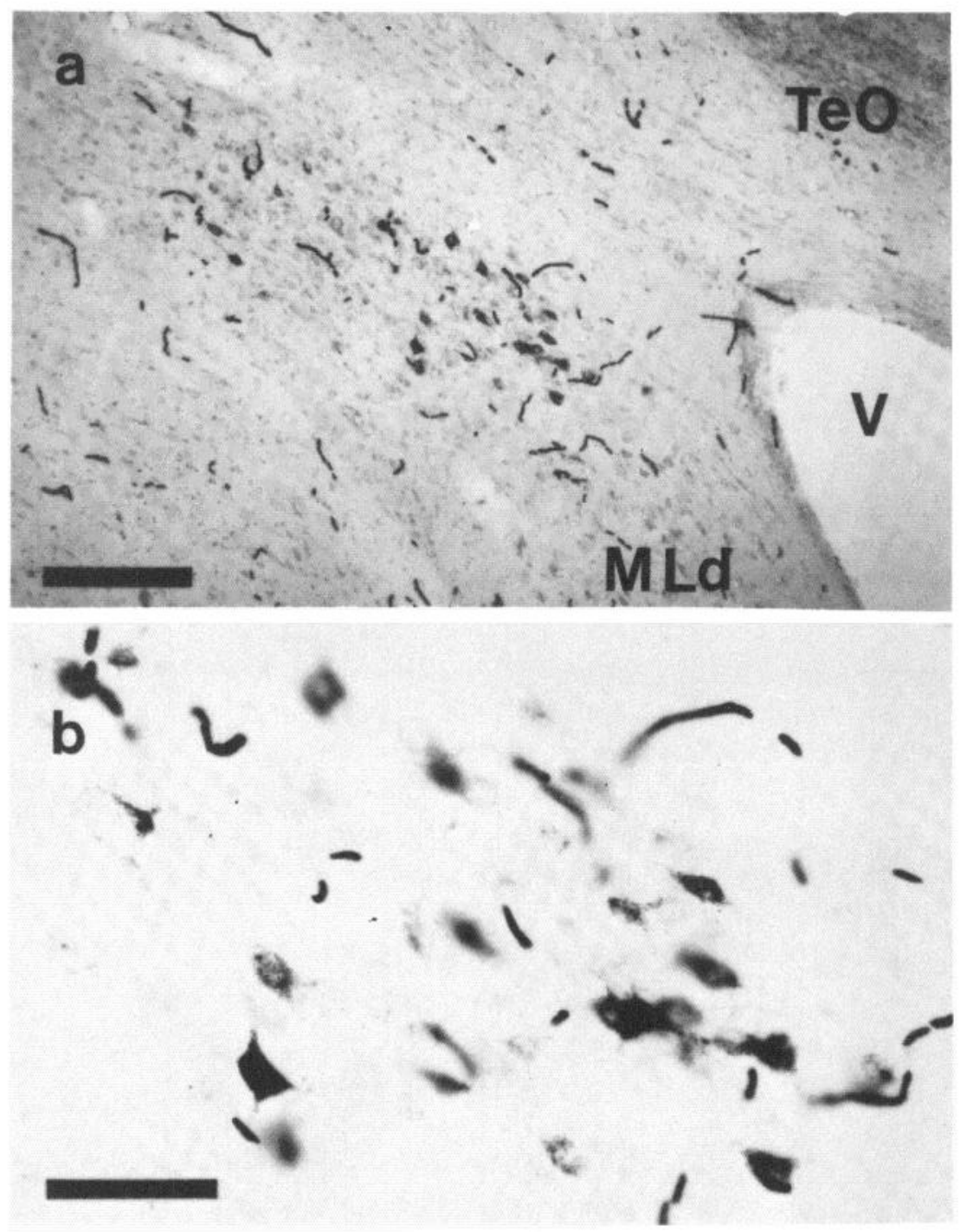

Figure 4. Photomicrographs of the dorsomedial nucleus of the nucleus intercollicularis (DM) at two different magnifications. $a$, View of the shape of $\mathrm{DM}$ as seen in a transverse section. Calibration bar $=200 \mu \mathrm{m}$. $b$, Higher magnification picture showing the morphology of labeled cells within DM. Calibration bar $=50 \mu \mathrm{m}$. 

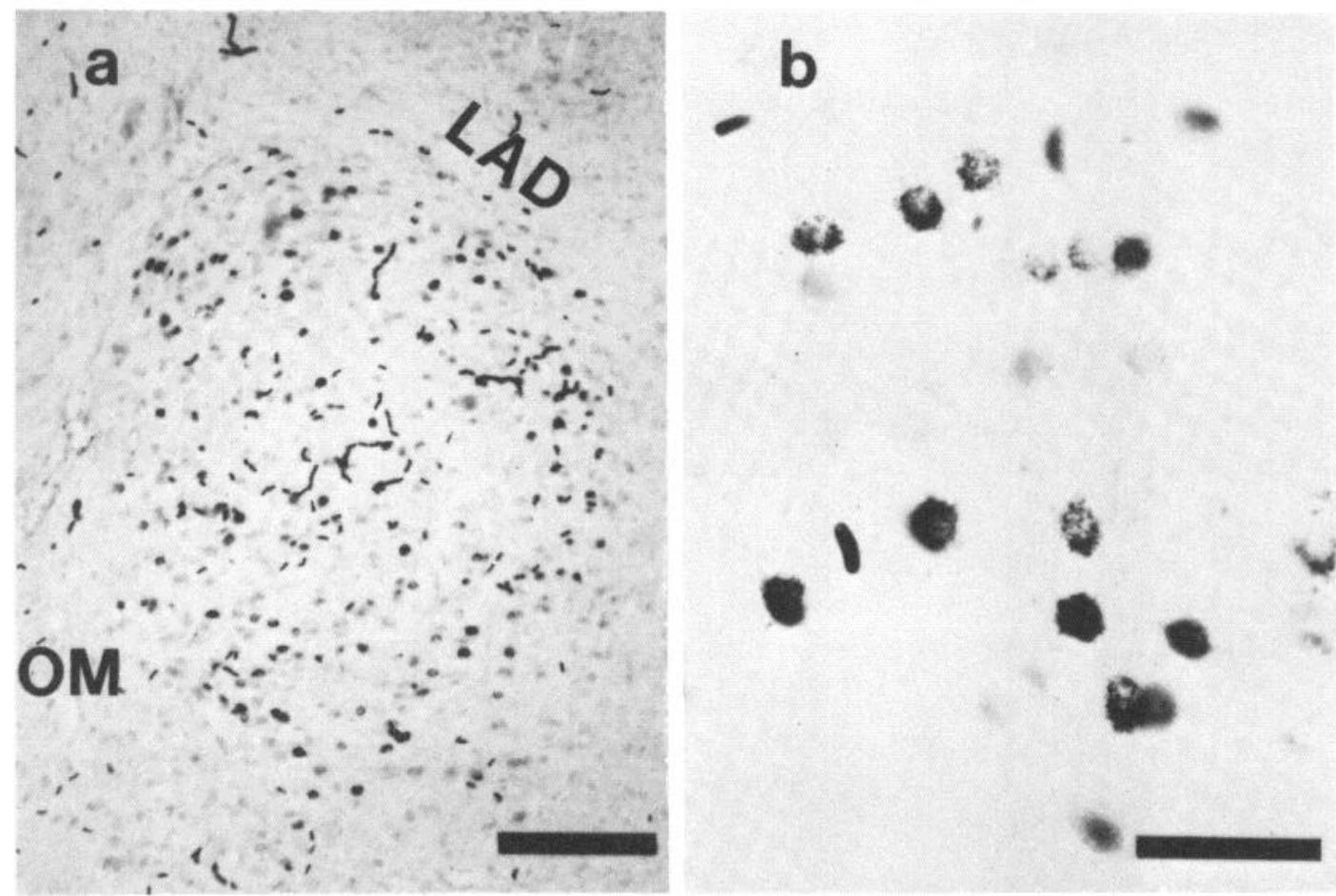

Figure 5. Photomicrographs of RA. a, View of the shape of RA as seen in transverse sections. Calibration bar $=200 \mu \mathrm{m}$. $b$, Higher magnification picture of labeled cells within RA. Calibration bar $=50 \mu \mathrm{m}$.

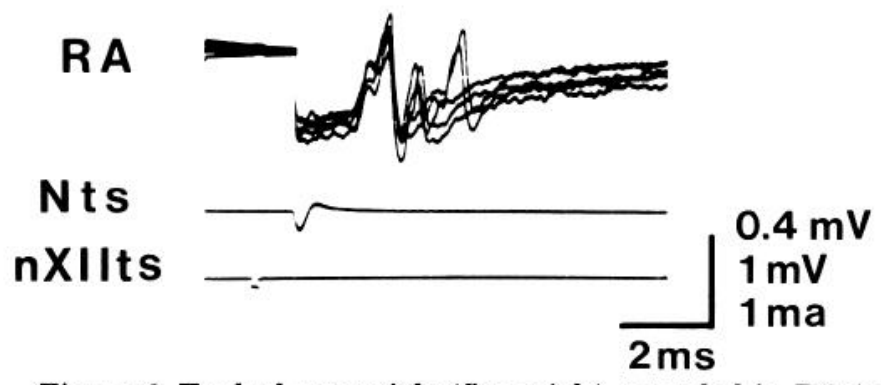

Figure 6. Evoked potentials (five trials) recorded in RA in response to antidromic stimulation of RA terminals in nXIIts.

cephalon labeled in the previous series of experiments. Typical antidromic responses in RA are shown in Figure 6. After mapping the boundaries of RA electrophysiologically, HRP (or tritiated proline in one case) was iontophoresed near the center of the nucleus. Subsequently, label was present from the brainstem to anterior neostriatum in the areas summarized in the reconstruction shown in Figure 7.

Caudal to the injection site, many labeled fibers were found in the occipitomesencephalic tract on the ipsilateral side, and fewer were seen in the anterior commissure and in the occipitomesencephalic tract of the contralateral side (Fig. $7 b$ ). In both HRP and tritiated prolineinjected preparations, labeled fibers were seen in ipsilateral DM (Fig. 7c). Labeled cells were found bilaterally in an area identified as the nucleus dorsointermedius posterior thalami based on similarities in position and shape to the nucleus of the same name in the canary (Stokes et al., 1974). Labeled fibers in the ipsilateral and, to a lesser extent, in the contralateral occipitomesencephalic tract

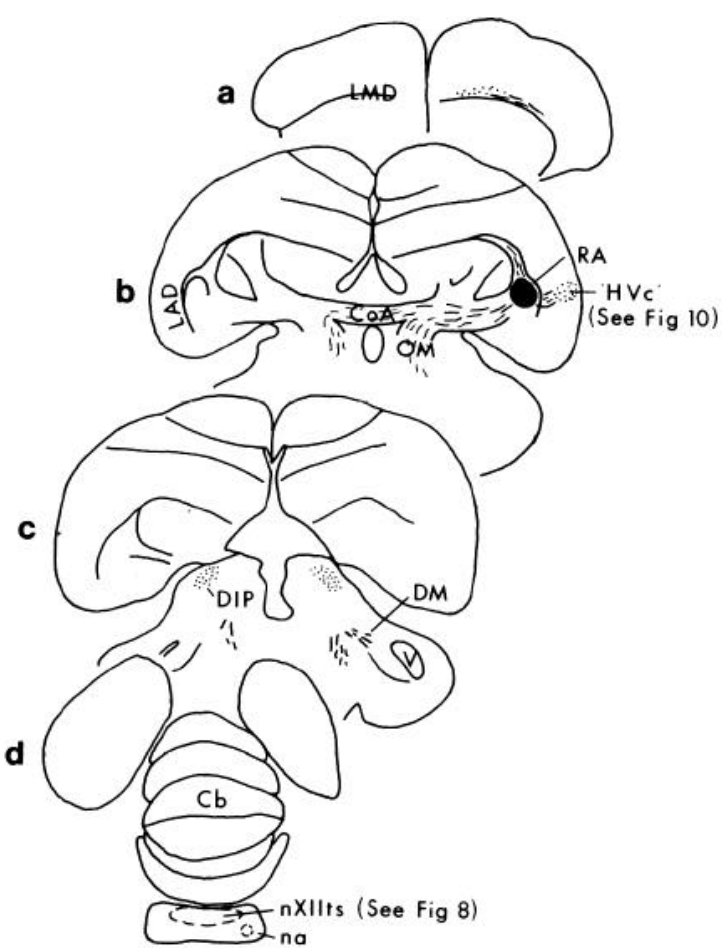

Figure 7. Reconstruction from transverse sections of the brain showing areas labeled after injection of HRP into RA. The sections are named from rostral $(a)$ to caudal $(d)$. The injection site is black, representative labeled fibers appear as lines, boundaries of terminal fields are shown as dashed lines, and representative labeled cells appear as dots. Photomicrographs of nXIIts and "HVc" from this brain are shown in Figures 8 and 10, respectively. 
continued caudally to the brainstem where they turned medially and entered nXIIts. Near nXIIts, fibers were labeled in the commissura infima. Within nXIIts, fibers branched extensively to form a dense feltwork of label equally distributed over both sides as shown in the darkfield pictures of Figure 8. No labeled cells were seen in nXIIts; thus, nXIIts appears to be a terminal field for RA axons. This is not surprising since the previous series of injections showed that RA cells were filled retrogradely by HRP in nXIIts. The important point is that the projection from each RA to the motor nucleus is bilateral so that either the left or right RA projects to motor neurons on both right and left nXIIts.

Another labeled terminal field was seen in the medulla ventrolateral to nXIIts (Figs. $7 d$ and 9). This area may represent a projection, albeit somewhat weaker, to the laryngeal motor neurons described by Manogue and Nottebohm (1981).

Rostral to the injection site in RA, fibers ran rostro- medially within lamina medullaris dorsalis to end above this Iamina in a sparse layer of labeled cells (Fig. 7a). Based on its position in the anterior neostriatum, its apposition to lamina medullaris dorsalis, and its projection to RA, we tentatively identified this area of labeled cells as the magnocellular nucleus of the anterior neostriatum.

Fibers also left RA laterally to enter a dense accumulation of labeled cells within the neostriatum (Figs. $7 b$ and 10). The cells within this area were multipolar with somas of 8 to $10 \mu \mathrm{m}$ (sample of 20 cells in one nucleus). Cells were labeled uniformly and heavily, in contrast to the granular label seen, for example, with cells of DM and RA in the first set of injections. While reserving final judgement on the identity of this area, we will tentatively refer to it as "HVc" since it may correspond to the nucleus hyperstriatum ventrale pars caudale in the canary.

Afferents and efferents of "HVc." In three birds, we
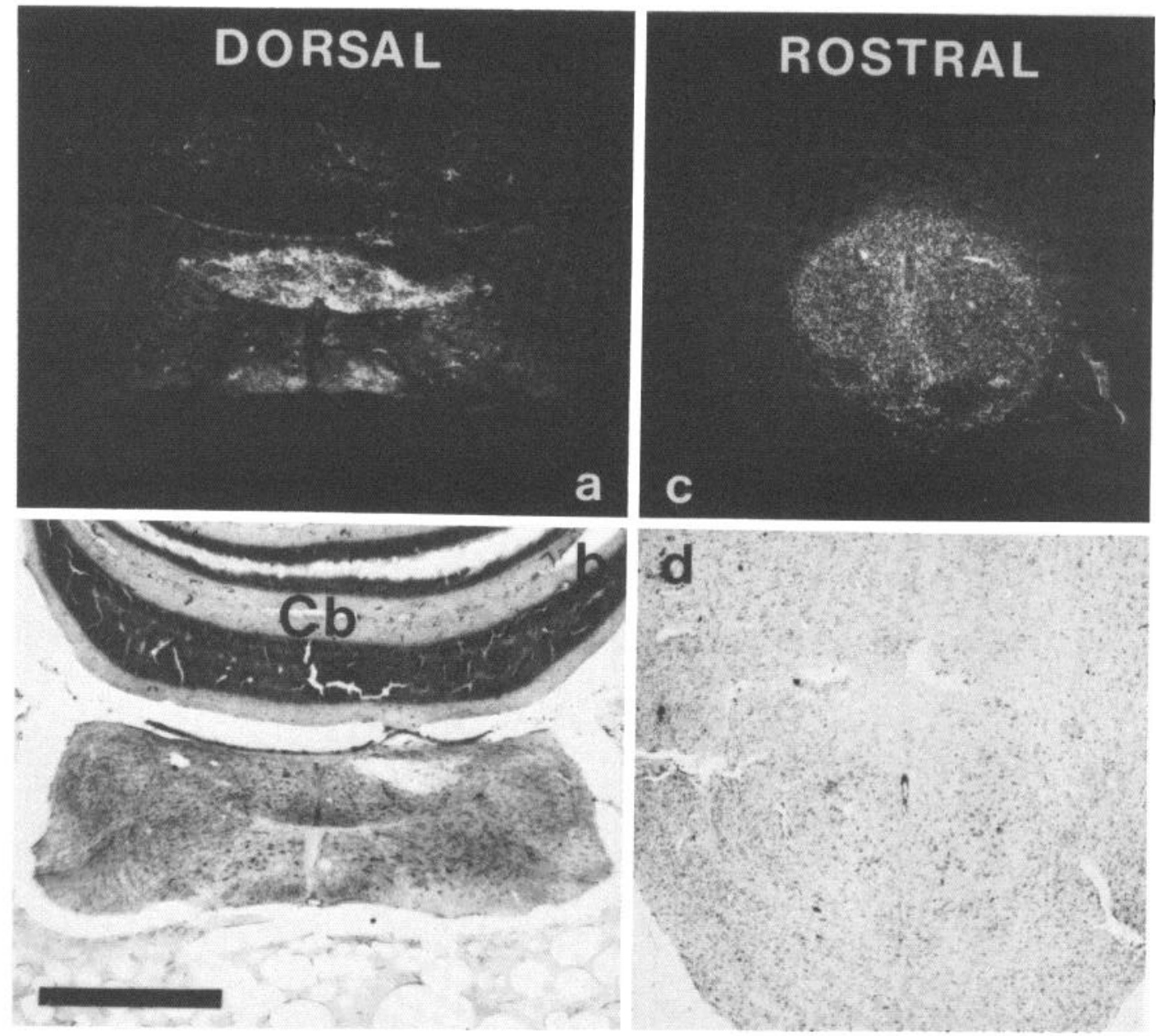

Figure 8. Photomicrographs of fibers from RA terminating within nXIIts. Calibration bar $=1$ $\mathrm{mm}$. $a$ and $b$ are dark-field and transmitted light pictures of the same transverse section through the medulla at the level of nXIIts. In the animal from which this section was taken (same brain as in Fig. 7), RA on one side was injected with HRP. HRP-labeled fibers appear over both sides of nXIIts as best seen in the dark-field picture. $c$ and $d$ are dark-field and transmitted light pictures of the same horizontal section through nXIIts. In the animal from which this section was taken, RA on one side was injected with tritiated proline. Autoradiographic label in the dark-field picture appears over both sides of nXIIts. 

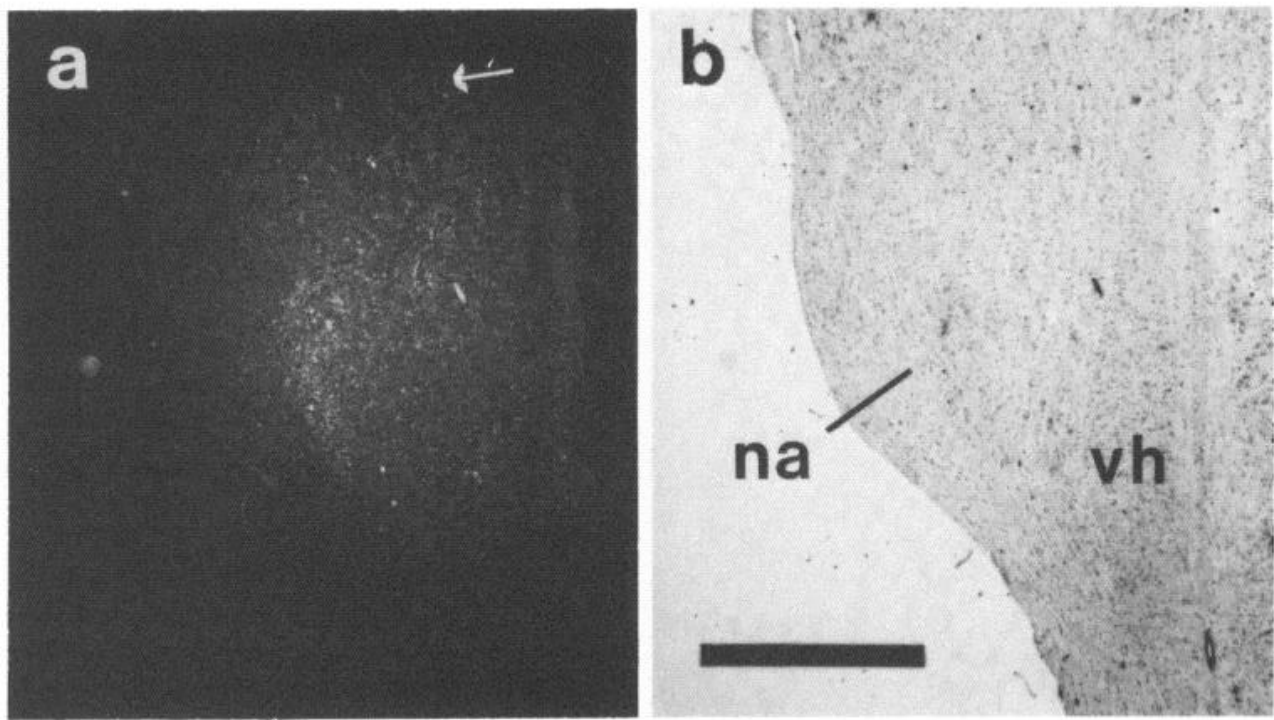

Figure 9. Photomicrograph of proline within the nucleus ambiguus as shown in a horizontal section at the level of ventral horn cells. $a$, Dark-field picture showing autoradiographic label; the arrow marks labeled fibers descending to the nucleus ambiguus and nXIIts. $b$, Light-field view of counterstained cells. Calibration bar $=1 \mathrm{~mm}$.
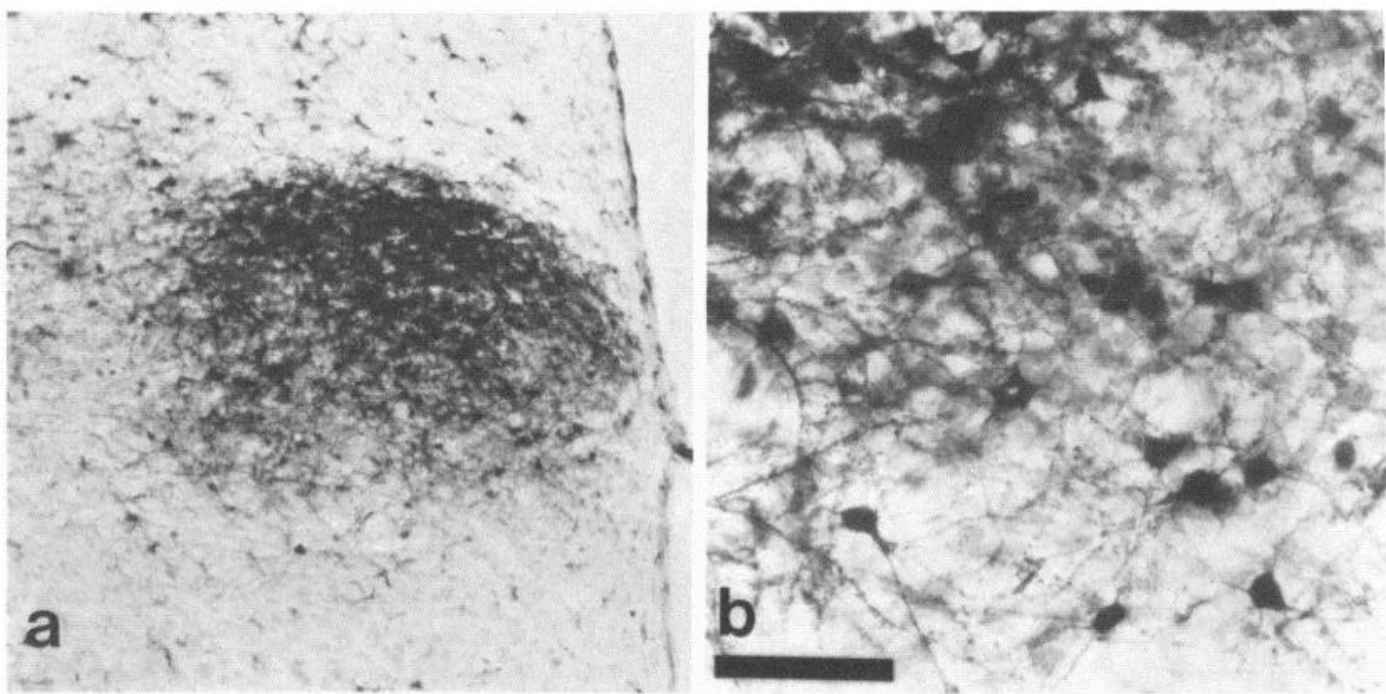

Figure 10. Photomicrographs of labeled cells in "HVc." $a$, View showing the shape of "HVc" in a transverse section. $b$, A higher magnification picture showing the morphology of labeled cells within "HVc." Calibration bar $=200 \mu \mathrm{m}$ in $a$ and $50 \mu \mathrm{m}$ in $b$.

injected HRP into "HVc" to test our identification of this area. A summary of the areas labeled is shown in Figure 11.

Labeled cells were found in the ipsilateral telencephalon, within the area which we have called the magnocellular nucleus of anterior neostriatum (Fig. 11a), and in an elongated neostriatal area which rises dorsally from lamina medullaris archistriatalis (Fig. 11a). Based on its shape, this second area may correspond to the nucleus interface (F. N. Nottebohm, D. B. Kelley, and J. A. Paton, submitted for publication) in canaries. Labeled cells also were found bilaterally in a small round nucleus within the thalamus (Fig. 11c). All of these labeled nuclei are comparable to areas which project to HVc in the canary. A few labeled cells also were found consistently in the habenula (Fig. 11d).

"HVc" itself projects to RA (Figs. $11 b$ and 12). Labeled fibers from the injection site entered RA and ramified there. There were no labeled cells in RA. The fiber terminals in RA indicate that the retrograde labeling of "HVc" cells in the previous set of injections was not due to interruption of fibers of passage near RA.

The anatomical projection of "HVc" then was confirmed physiologically by microstimulation. When either ipsilateral or contralateral "HVc" was stimulated electrically with a train of cathodal pulses, action potentials appeared in the tracheosyringeal nerve with a latency of 12 to 15 msec (Fig. 13). 


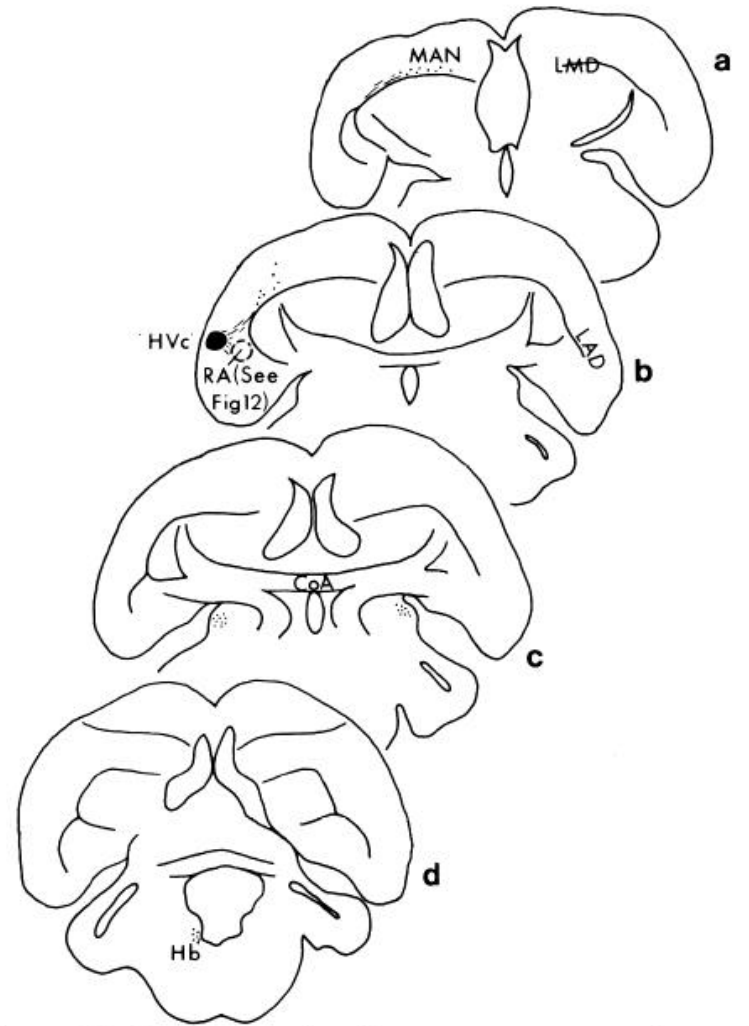

Figure 11. Reconstruction from transverse sections of the brain showing areas labeled after the injection of HRP into "HVc." Sections are labeled from rostral $(a)$ to caudal $(d)$. The injection site is black, representative labeled fibers appear as lines, terminal fields are bounded by dashed lines, and representative labeled cells are shown as dots. A photomicrograph of the terminal field in RA is shown in Figure 12.

All of the preceding observations on afferents, efferents, and physiology support our identification of " $\mathrm{HVc}$ " as being comparable to HVc in the canary.

\section{Discussion}

Vocal control nuclei. We found the vocal pathway of budgerigars to consist of (1) a neostriatal nucleus, "HVc;" (2) an archistriatal nucleus, RA; (3) a mesencephalic nucleus, DM; and (4) the medullary nucleus, nXIIts (Fig. 14). These nuclei are similar in cell morphology, gross structure, afferents, and efferents to vocal control nuclei in the canary (Nottebohm et al., 1976). The absolute positions are, however, quite different; for example, HVc in canaries is in dorsomedial telencephalon, while "HVc" in budgerigars is adjacent to the lateral edge of the telencephalon. On the other hand, positions relative to adjacent nuclei and fiber tracts appear to be the same in both species, as if the brain had been transformed topologically so as to maintain connections but to change the absolute positions. Thus, the relatively large telencephalon of the budgerigar (as noted by Portman and Stingelin, 1961) appears to have pushed the vocal control nuclei laterally.

We have used the same names for comparable nuclei in budgerigars and canaries with the exception of "HVc" which, in budgerigars, is clearly within the neostriatum and not the hyperstriatum. In canaries, HVc is bounded

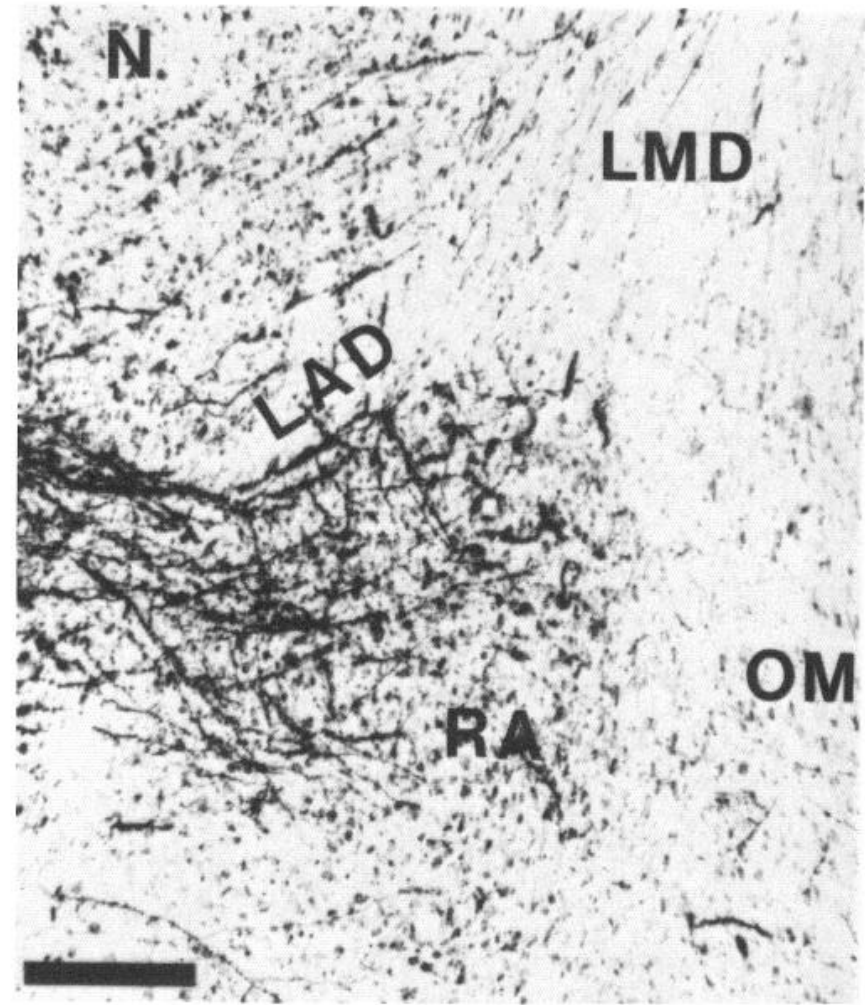

Figure 12. Photograph of HRP-labeled fibers from "HVc" terminating in RA. Medial is to the right and dorsal is at the top. Calibration bar $=200 \mu \mathrm{m}$.

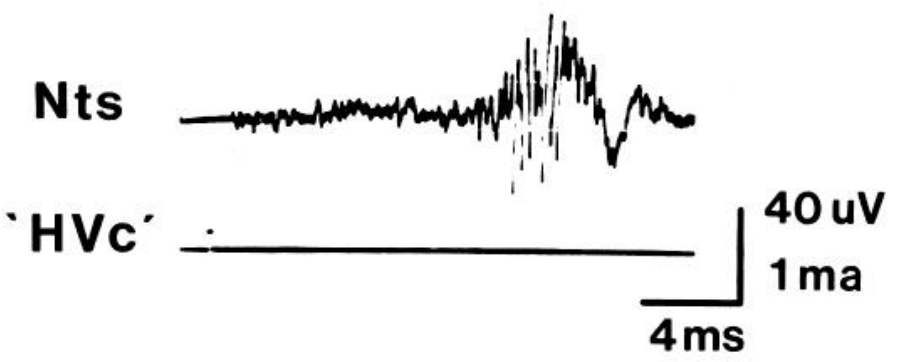

Figure 13. Whole nerve recording from Nts showing the response to electrical stimulation of contralateral "HVc." The stimulus was one of a train of pulses presented at 50 -msec intervals.

ventrally by a well defined lamina which had been identified previously in transverse sections as the most caudal extent of the lamina hyperstriatica, and thus, the nucleus was thought to be in the hyperstriatum. After a reexamination of this same area in sagittal sections, we now believe that HVc in canaries is actually in the neostriatum as it is in budgerigars.

HVc and RA have been recognized as cytoarchitectonically discrete nuclei in a variety of oscine songbirds (see Nottebohm (1980) p. 118, for a summary), but using canary anatomy as a model, they have gone undetected both in non-oscine Passeriformes and in Columbiformes, such as the pigeon, which lack vocal learning. If, on the other hand, the budgerigar brain is used as a model, a reasonable candidate for RA in the pigeon is archistriatum pars ventralis (Karten and Hodos (1967) p. 79, 


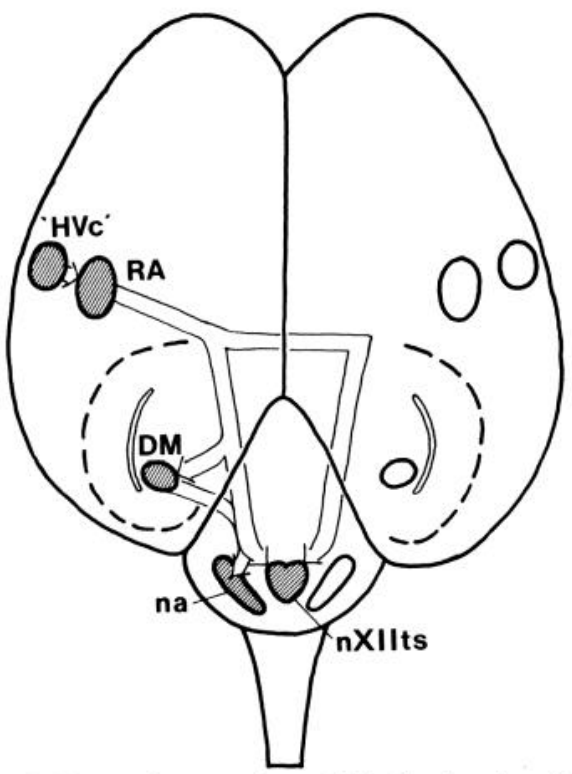

Figure 14. Schematic top view of the budgerigar brain showing a summary of the vocal pathway. To simplify this figure, the fibers from RA which cross the midline dorsal to nXIIts in the commissura infima are not shown.

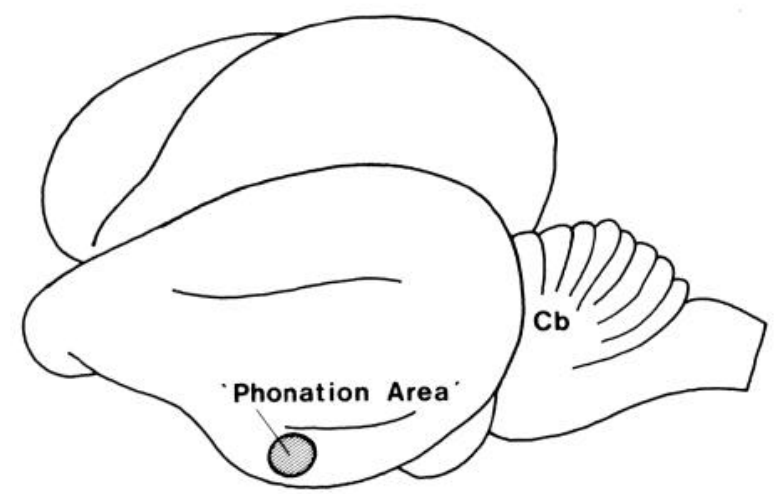

Figure 15. Schematic side view of parrot brain, after Figure 7 in Kalischer (1905). The "phonation area" was determined by lesion and faradic stimulation experiments.

A7.50). Archistriatum pars ventralis, later renamed archistriatum intermedium, receives its input from the overlying neostriatum and/or hyperstriatum by way of the tractus archistriatalis dorsalis and projects to the brainstem and rostral spinal cord by way of the occipitomesencephalic tract (Zeier and Karten, 1971). These tracts are the same as those to and from RA in the canary; it remains to be seen whether specific connections to nXIIts and the equivalent of HVc are present in the pigeon. This information on vocal circuitry in species without vocal learning could potentially narrow the search for neural substrates of vocal learning.

Vocal control pathway. Although the vocal nuclei in budgerigars and canaries are similar, their connections differ. In canaries, the projections from $\mathrm{HVc}$ to RA to nXIIts are all strictly ipsilateral (Nottebohm et al., 1976). In budgerigars, the projections from RA to nXIIts and from nXIIts to the syrinx are bilateral (this paper; Manogue and Nottebohm, 1981).

The projection from RA to nXIIts appears to decussate at two locations. First, fibers from RA cross the midline in the anterior commissure to enter the contralateral occipitomesencephalic tract. In both HRP and proline experiments, the contralateral occipitomesencephalic tract contained sustantially fewer labeled fibers than the ipsilateral occipitomesencephalic tract, yet label appeared to be distributed uniformly over both halves of nXIIts (Fig. 8). One explanation for this difference is that fibers from RA to nXIIts also cross the midline near nXIIts in the commisura infima, which did, in fact, contain labeled fibers. We do not know yet whether separate sets of RA neurons project to opposite sides of the motor nucleus or whether collaterals of RA cells project bilaterally. It should be possible to answer this question using any of the newly developed fluorescent, retrograde double labeling techniques (e.g., Van der Kooy and Kuypers, 1979) or antidromic stimulation methods (e.g., Shinoda et al., 1976).

The motor nerves from nXIIts cross the midline in an anastomosis above the syrinx. In this projection, anatomical evidence exists for the formation of collaterals within the anastomosis (Manogue and Nottebohm, 1981).

What is the functional significance of the bilateral projections in the budgerigar? As a result of its bilateral projections, $\mathrm{RA}$ - and $\mathrm{HVc}$, in turn - can potentially control the activity of both sides of nXIIts. In fact microstimulation of either RA or HVc led to activity in both ipsilateral and contralateral tracheosyringeal nerves. What effect will this have on the possibility of hemispheric dominance? In terms of gross connectivity, each RA duplicates what the other RA can do. To function differently then, each RA would have to have access to different kinds of information or different triggering inputs; that is, the role of "HVc" should differ between the two sides. This we are now ready to explore.

If the vocal control nuclei in the budgerigar are truly homologous to those in the canary, it would be tempting to speculate that the species differences in projections, unilateral versus bilateral, might represent one mode of evolution of the nervous system. That is, nuclei are conserved, while connections between nuclei change. Certainly genetically determined differences in the degree of pathway crossing occur in other vertebrate pathways, notably in the visual system (reviewed in Lund (1978) pp. 138-165) and in the nigrotectal projection (Beckstead et al., 1981). The budgerigar/canary differences represent a particularly striking, and possibly more experimentally tractable, system to add to this list. In other systems, such as the isthmo-optic nucleus, collateral projections are formed during early development and are later retracted (reviewed in Cowan and Clarke, 1976). Does the same thing occur here; that is, do canaries begin with bilateral connections which they later lose? Alternatively, is there some factor present in budgerigars which favors decussation or the formation of collateral projections, such as those which can occur due to lesions (e.g., Goldman, 1978)? Are the bilateral projections of $\mathrm{RA}$ and nXIIts related or independent during development?

Finally, a historical comment seems appropriate. In 1905 , Kalischer tried to demonstrate the localization of vocal control in parrots, also psittaciforms. Influenced by 
the then recent findings of Paul Broca, Karl Wernicke, and their followers, Kalischer placed lesions on the lateral surface of the brain, an area that he thought would be homologous to the temporal lobe of humans (see Fig. 15). Such lesions did disrupt vocal behavior (Kalischer, 1905). In retrospect, it seems as if Kalischer's lesions probably damaged both RA and "HVc." Had Kalischer placed his lesions in the "temporal lobe" of canaries, his efforts at testing the teachings of Broca would have failed, but then would this have been surprising? After all, canaries do not talk.

\section{References}

Adams, J. C. (1977) Technical considerations on the use of horseradish peroxidase as a neuronal marker. Neuroscience 2: 141-145.

Adams, J. C. (1980) Stabilizing and rapid thionin staining of TMB-based HRP reaction product. Neurosci. Lett. 17: 7-9.

Beckstead, R. M., S. B. Edwards, and A. Frankfurter (1981) A comparison of the intranigral distribution of nigrotectal neurons labeled with horseradish peroxidase in the monkey, cat, and rat. .J. Neuroscience 1: 121-125.

Cowan, W. M., and P. G. H. Clarke (1976) The development of the isthmo-optic nucleus. Brain Behav. Evol. 13: 345-375.

Gazzaniga, M. S. (1970) The Bisected Brain, Appleton-Century-Crofts, New York.

Gazzaniga, M. S., and J. E. LeDoux (1978) The Integrated Mind, Plenum, New York.

Geschwind, N. (1970) The organization of language and the brain. Science 170: $940-944$.

Goldman, P. (1978) Neuronal plasticity in primate telencephalon: Anomalous projections induced by prenatal removal of frontal cortex. Science 202: 768-770.

Gurney, M. E., and M. Konishi (1979) A cellular analysis of androgen- and estrogen-induced sexual differentiation in the zebra finch song system. Soc. Neurosci. Abstr. 5: 446.

Kalischer, O. (1905) Das grosshirn der Papageien in anatomischer und physiologischer Bezeihung. Abh. Preuss. Akad. Wiss. IV 1: 1-105.

Karten, H. J., and W. Hodos (1967) A Stereotaxic Atlas of the Brain of the Pigeon (Columba livia), The Johns Hopkins University Press, Baltimore.

Kelley, D. B., and F. Nottebohm (1979) Projections of a telencephalic auditory nucleus-Field L-in the canary. J. Comp. Neurol. 183: 455-470.

Lemon, R. E. (1973) Nervous control of the syrinx in whitethroated sparrows (Zonotrichia albicolis). J. Zool. (Lond.) 171: $131-140$.

Lund, R. D. (1978) Development and Plasticity of the Brain, An Introduction, Oxford University Press, New York.

Malmgren, L., and Y. Olsson (1978) A sensitive method for histochemical demonstration of horseradish peroxidase in neurons following retrograde axonal transport. Brain Res. 148: 279-294.

Manogue, K. R., and F. Nottebohm (1981) Relation of medullary efferent nuclei to nerves supplying the vocal tract of the budgerigar (Melopsittacus undulatus). J. Comp. Neurol., in press.

Mesulam, M. -M. (1978) Tetramethyl benzidine for horseradish peroxidase neurochemistry: A non-carcinogenic blue reaction product with superior sensitivity for visualizing neural afferents and efferents. J. Histochem. Cytochem. 26: 106-117.

Milner, B. (1974) Hemispheric specialization: Scope and limits. In The Neurosciences: Third Study Program, F. O. Schmitt and F. G. Worden, eds., pp. 75-89, The MIT Press, Cambridge, MA.

Nottebohm, F. (1971) Neural lateralization of vocal control in a passerine bird. I. Song. J. Exp. Zool. 177: 229-261.

Nottebohm, F. (1976) Phonation in the orange-winged Amazon parrot, Amazona amazonica. J. Comp. Physiol. A Sens. Neural Behav. Physiol. 108: 157-170.

Nottebohm, F. (1977) Asymmetries in neural control of vocalization in the canary. In Lateralization in the Nervous System, S. K. Harnad, ed., pp. 23-44, Academic Press, New York.

Nottebohm, F. (1979) Origins and mechanisms in the establishment of cerebral dominance. In Handbook of Behavioral Neurobiology, M. S. Gazzaniga, ed., Vol. 2, pp. 295-344, Plenum, New York.

Nottebohm, F. (1980) Brain pathways for vocal learning in birds: A review of the first 10 years. Prog. Psychobiol. Physiol. Psychol. 9: 85-124.

Nottebohm, F., and M. Nottebohm (1976) Left hypoglossal dominance in the control of canary and white-crowned sparrow song. J. Comp. Physiol. A Sens. Neural Behav. Physiol. 108: 171-192

Nottebohm, F., T. M. Stokes, and C. M. Leonard (1976) Central control of song in the canary, Serinus canarius. J. Comp. Neurol. 165: 457-486.

Paton, J. A., K. R. Manogue, and F. Nottebohm (1980) Telencephalic efferent pathways for vocal control in parakeets. Soc. Neurosci. Abstr. 6: 587.

Portman, A., and W. Stingelin (1961) The central nervous system. In Biology and Comparative Physiology of Birds, A. J. Marshall, ed., Vol. II, Chap. XIII, pp. 1-36, Academic Press, New York.

Seller, T. J. (1979) Unilateral nervous control of the syrinx in Java sparrows. J. Comp. Physiol. A Sens. Neural Behav. Physiol. 129: 281-288.

Shinoda, Y., A. Arnold, and H. Asanuma (1976) Spinal branching of corticospinal axons in the cat. Exp. Brain Res. 26: 215234.

Sperry, R. W. (1974) Lateral specialization in the surgically separated hemispheres. In The Neurosciences: Third Study Program, F. O. Schmitt and F. G. Worden, eds., pp. 5-19, MIT Press, Cambridge, MA.

Stokes, T. M., C. M. Leonard, and F. Nottebohm (1974) The telencephalon, diencephalon, and mesencephalon of the canary, Serinus canarius, in stereotaxic coordinates. J. Comp. Neurol. 156: 337-374.

Van der Kooy, D., and H. G. J. M. Kuypers (1979) Fluorescent retrograde double labeling: Axonal branching in the ascending raphe and nigral projections. Science 204: 873-875.

Zeier, H., and H. J. Karten (1971) The archistriatum of the pigeon: Organization of the efferent and afferent connections. Brain Res. 31: 313-326. 